\title{
Gravity Probe B: Final Results of a Space Experiment to Test General Relativity
}

\author{
C. W. F. Everitt, ${ }^{1, *}$ D. B. DeBra, ${ }^{1}$ B. W. Parkinson, ${ }^{1}$ J. P. Turneaure, ${ }^{1}$ J. W. Conklin, ${ }^{1}$ M. I. Heifetz, ${ }^{1}$ G. M. Keiser, ${ }^{1}$ \\ A. S. Silbergleit, ${ }^{1}$ T. Holmes, ${ }^{1}$ J. Kolodziejczak, ${ }^{2}$ M. Al-Meshari, ${ }^{3}$ J. C. Mester, ${ }^{1}$ B. Muhlfelder, ${ }^{1}$ V. G. Solomonik, ${ }^{1}$ \\ K. Stahl, ${ }^{1}$ P. W. Worden, Jr., ${ }^{1}$ W. Bencze, ${ }^{1}$ S. Buchman, ${ }^{1}$ B. Clarke, ${ }^{1}$ A. Al-Jadaan, ${ }^{3}$ H. Al-Jibreen, ${ }^{3}$ J. Li, ${ }^{1}$ J. A. Lipa, ${ }^{1}$ \\ J. M. Lockhart, ${ }^{1}$ B. Al-Suwaidan, ${ }^{3}$ M. Taber, ${ }^{1}$ and S. Wang ${ }^{1}$ \\ ${ }^{1}$ HEPL, Stanford University, Stanford, California 94305-4085, USA \\ ${ }^{2}$ George C. Marshall Space Flight Center, Huntsville, Alabama 35808, USA \\ ${ }^{3}$ King Abdulaziz City for Science and Technology, Riyadh, Saudi Arabia
}

(Received 1 April 2011; published 31 May 2011)

Gravity Probe B, launched 20 April 2004, is a space experiment testing two fundamental predictions of Einstein's theory of general relativity (GR), the geodetic and frame-dragging effects, by means of cryogenic gyroscopes in Earth orbit. Data collection started 28 August 2004 and ended 14 August 2005. Analysis of the data from all four gyroscopes results in a geodetic drift rate of $-6601.8 \pm$ $18.3 \mathrm{mas} / \mathrm{yr}$ and a frame-dragging drift rate of $-37.2 \pm 7.2 \mathrm{mas} / \mathrm{yr}$, to be compared with the GR predictions of $-6606.1 \mathrm{mas} / \mathrm{yr}$ and $-39.2 \mathrm{mas} / \mathrm{yr}$, respectively ("mas" is milliarcsecond; 1 mas $=$ $\left.4.848 \times 10^{-9} \mathrm{rad}\right)$.

DOI: 10.1103/PhysRevLett.106.221101

PACS numbers: 04.80.Cc

Introduction.-In 1960, Schiff [1] showed that an ideal gyroscope in orbit around Earth would undergo two relativistic precessions with respect to a distant inertial frame: (1) a geodetic drift in the orbit plane due to motion through the space-time curved by Earth's mass and (2) a frame-dragging due to Earth's rotation. The geodetic term matches the curvature precession of the Earth-Moon system around the Sun given by de Sitter in 1916 [2]. The Schiff frame dragging is related to the dragging of the orbit plane of a satellite around a rotating planet computed by Lense and Thirring in 1918 [3]. Frame dragging has important implications for astrophysics; it has been invoked as a mechanism to drive relativistic jets emanating from galactic nuclei [4].

The measurement requires one or more gyroscopes referenced to a remote star by an onboard telescope. In the $642 \mathrm{~km}$ polar orbit of Gravity Probe B (GP-B), the two effects are at right angles, as in Fig. 1. The predicted geodetic drift rate is $-6606.1 \mathrm{mas} / \mathrm{yr}$; the frame-dragging drift rate with the chosen star IM Pegasi is $-39.2 \mathrm{mas} / \mathrm{yr}$.

GP-B was conceived as a controlled physics experiment having submas/yr stability $\left(10^{6}\right.$ times better than the best modeled navigation gyroscopes) with numerous built-in checks and methods of treating systematics. Three principles guided the design: (1) make Newtonian gyro drifts much less than the predicted GR effects; (2) add sensors so that modeling hinges on physical understanding as against the ad hoc observational modeling used in navigation gyroscopes; (3) exploit natural effects such as stellar aberration in calibrating the instrument. Meeting the many mechanical, optical, and electrical requirements rested on a conjunction of two technologies, space and cryogenics.

Operation in space separates the two effects, increases the geodetic effect 12.4 times as compared to a gyroscope at the equator, eliminates "seeing" in the measurement to the guide star, and vastly reduces torques from suspending the gyroscope against $1 g$ gravity. Cryogenics brings new levels of magnetic shielding, thermal isolation, ultrahigh vacuum operation, and a uniquely effective gyro readout based on the London moment in a spinning superconductor. The two together give the instrument ultimate mechanical stability: in zero $g$, there is no sag; at zero $\mathrm{K}$, there is no thermal distortion.

Essential to GP-B as a controlled physics experiment was the calibration phase, a 46-day period following the main science phase designed to set limits on a range of potential disturbing effects and quantify any that might prove larger than expected.

Experiment description.-The heart of the instrument was a $0.92 \mathrm{~m}$ long fused silica structure containing four gyroscopes and a star-tracking telescope mounted in a 24401 superfluid helium Dewar operating at $1.8 \mathrm{~K}$. Each

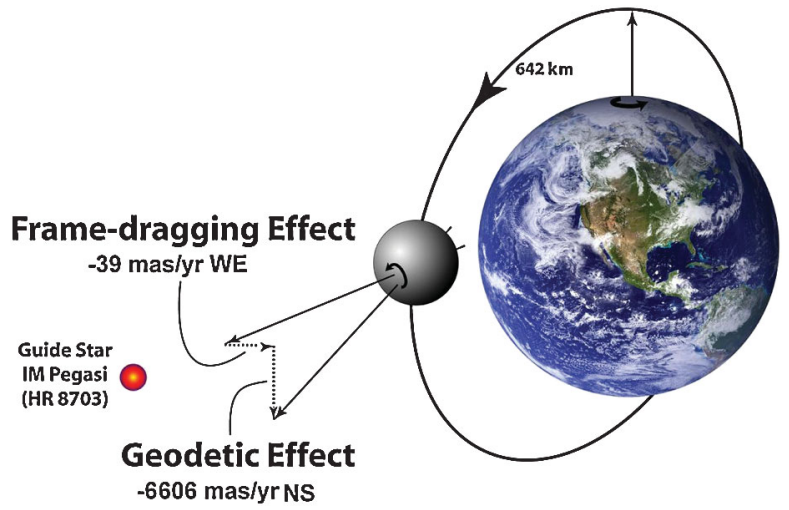

FIG. 1 (color). Predicted drift rates of GP-B gyroscopes. See [17] for definitions of WE and NS inertial directions. 
gyroscope comprised a $38 \mathrm{~mm}$ diameter niobium-coated fused quartz sphere suspended electrically, spun up by helium gas, and read out magnetically. The four were set in line, two spinning clockwise and two counterclockwise, with axes initially aligned to the boresight of the telescope. The space vehicle rolled with $77.5 \mathrm{~s}$ period about the line of sight to IM Pegasi, which was, however, occulted by Earth for almost half each orbit (Fig. 1). During occulted periods, pointing was referenced to star trackers and rate gyros on the outside of the spacecraft. Drag compensation, originated by Pugh in an independent proposal for an orbiting gyroscope experiment [5], two months prior to Schiff's Letter, was by a control system referred to one of the gyroscopes as a proof mass. Attitude, translational, and roll control authority was provided by the helium boil-off gas from the Dewar vented through proportional thrusters [6].

A gyro readout that did not disturb the spin orientation was vital. Superconductivity supplied three essentials. The spinning rotor generated a London moment equivalent at $80 \mathrm{~Hz}$ to a uniform $5 \times 10^{-5} \mathrm{G}$ field aligned with the spin axis. A SQUID magnetometer coupled to a superconducting loop on the gyro housing provided a readout capable of detecting a 1 mas change of spin direction in $10 \mathrm{~h}$. Finally, a combination of high permeability and ultralow field superconducting shields around the instrument with local shields for each gyroscope achieved (1) $240 \mathrm{~dB}$ isolation from external magnetic disturbances, (2) a limit on trapped fields in the rotors $\sim 1 \%$ of the London moment, and (3) virtual elimination of magnetic torques.

The gyro readout scale factor $C_{g}(t)$ was calibrated on orbit against the aberration of starlight. During each halforbit when IM Pegasi was visible, the telescope pointed at its apparent position, and the 20.49586 arcsec annual and 5.18560 arcsec orbital aberrations, derived, respectively, from the JPL Earth ephemeris and GPS orbit data, appeared in the gyro readout.

An elementary calculation captures the stringencies of the experiment. Consider a spherical, not quite homogeneous rotor under transverse acceleration $f$. Let $\delta r$ separate the centers of mass and support, $v_{s} \sim 9 \mathrm{~m} / \mathrm{s}$ be the rotor's peripheral velocity, $\Omega_{0}$ the maximum allowed drift rate $0.1 \mathrm{mas} / \mathrm{yr}\left(1.5 \times 10^{-17} \mathrm{rad} / \mathrm{s}\right)$. Then, we have $\delta r / r<2 v_{s} \Omega_{0} / 5 f$, and mass unbalance requirements $\delta r / r: 6 \times 10^{-18}$ on Earth, $6 \times 10^{-10}$ in a typical $642 \mathrm{~km}$ altitude satellite, and $6 \times 10^{-7}$ for GP-B in $10^{-11} \mathrm{~g}$ dragfree mode. Without drag compensation, the experiment would have been impossible. Likewise for an aspherical homogeneous rotor, the electrical support torques, while greatly reduced on orbit, only reached the desired level through a further symmetrizing factor, spacecraft roll. The net allowed asphericity was $55 \mathrm{~nm}$.

Aiming for a $\sim 0.5 \mathrm{mas} / \mathrm{yr}$ mission, we created an error tree setting limits on 133 disturbing terms, and verified in advance that all were negligible. Seven "near zeros" (Table I) were central. Four noted already are inhomoge-
TABLE I. The seven near zeros.

\begin{tabular}{lcc}
\hline \hline Property & Requirement & Achieved \\
\hline Rotor properties & & \\
Mass unbalance, $\delta r / r$ & $6 \times 10^{-7}$ & $2-5 \times 10^{-7}$ \\
Asphericity $(\mathrm{nm})$ & 55 & $<33$ \\
Patch dipole $(\mathrm{C} \cdot \mathrm{m})$ & $<10^{-15}$ & see text \\
Environment & & \\
Cross-track acceleration $(g)$ & $10^{-11}$ & $10^{-11}$ \\
Gas pressure (torr) & $10^{-11}$ & $<10^{-14}$ \\
Rotor trapped field $(\mu \mathrm{G})$ & 9 & $0.2-3$ \\
Mixed & & \\
Rotor electric charge $(\mathrm{electrons})$ & $10^{8}$ & $<10^{8}$ \\
\hline \hline
\end{tabular}

neity and asphericity for the rotor, residual acceleration and magnetic field for the environment. The others are gyro electric charge, gas pressure, and patch effect. Six met requirements; the complication discovered during the calibration phase was the patch effect. Even so, the idea of seeing relativity in the "raw" data was preserved. Figure 2 shows the north-south (NS) drifts of the four gyroscopes with no torque modeling. The geodetic drift is visible in all four.

The term "patch effect" $[7,8]$ refers to contact potential differences between crystalline or contamination regions on a metal surface. Prelaunch studies focused on eddy current losses and torques from interaction of the rotor's patch-induced electric dipole moment with support voltages and the housing dipole moment. Far more important was the on-orbit discovery of forces and torques caused by interacting patches on the rotor and housing. Put simply, rotor and housing were spherical mechanically; electrically, they were not.

Three unforeseen effects emerged, differing in detail for each gyroscope: a changing polhode path and two patch effect torques. The changing polhode, originating in a $<1 \mathrm{pW}$ dissipation of the rotor's kinetic energy, complicated the $C_{g}$ determination. Beginning with their body axes arbitrarily oriented and a $2-6 \mathrm{~h}$ period, the rotors transitioned to a final $1-4 \mathrm{~h}$ period, each spinning nearly about

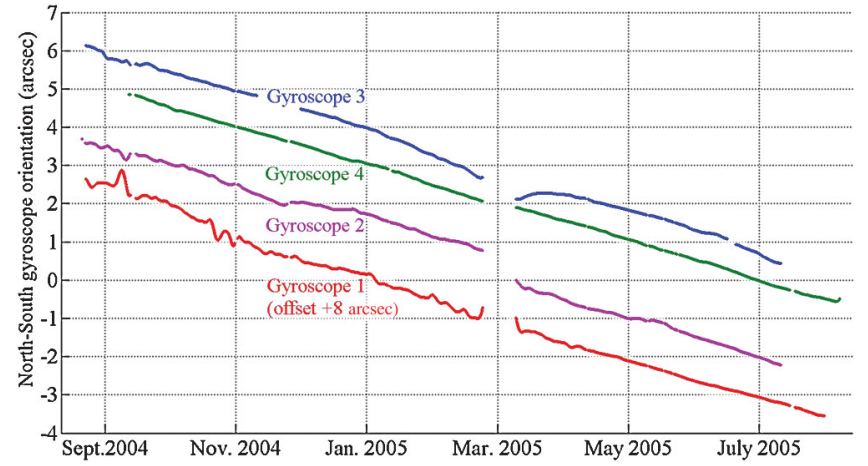

FIG. 2 (color). North-south gyro orientation histories with no modeling of torque or scale factor. 
its maximum inertia axis. The two torques were (1) a spin-to-roll misalignment torque $200-500 \times$ larger than predicted from mechanical asphericity, and (2) a "rollpolhode resonance" torque, where the roll averaging mentioned above would temporarily fail, making a particular gyroscope axis realign, or step over, by as much as 100 mas in 1-2 days when a high harmonic of its polhode rate came into resonance with spacecraft roll. The patch effect misalignment torque was discovered and quantified during the calibration phase by commanding the spacecraft to point to a series of positions at known angles to the guide star.

Remarkably, a key to modeling all three effects was magnetic asphericity: two patterns, magnetic fluxons and voltage patches, remained locked together in the rotor. A process of trapped flux mapping (TFM) allowed exact tracking of the evolving polhode phase and angle needed for computing both $C_{g}(t)$ and torques.

Further extensive and diversified postscience calibrations showed no significant disturbing effects other than the three just discussed.

Data analysis.-Two data analysis methods were used to determine and cross-check the relativity results. One, called "algebraic," was based on a parameter estimator utilizing the gyro dynamics and measurement models detailed below. It produced the primary science results (see Tables II and III, and Fig. 3). The other method, called "geometric," though currently less precise, neatly eliminated the need to model the misalignment torque. This torque, being Newtonian, causes drift rates perpendicular to the misalignment vector $\vec{\mu}$. The drift in that direction is thus a combination of relativistic and Newtonian contributions, while the drift rate parallel to $\vec{\mu}$ - when there is no roll-polhode resonance-is pure relativity. The annual variation in misalignment direction allows determination of both relativity effects.

Gyro dynamics. The $r_{\mathrm{NS}}$ (geodetic) and $r_{\mathrm{WE}}$ (framedragging) drift rates and two patch effect torques [9] connect to the unit vector $\hat{s}(t)$ along the gyro spin axis by the equations of motion:

$$
\begin{aligned}
& \dot{s}_{\mathrm{NS}}=r_{\mathrm{NS}}+k(t) \mu_{\mathrm{WE}}+\sum_{m}\left(a_{m} \cos \Delta \phi_{m}-b_{m} \sin \Delta \phi_{m}\right), \\
& \dot{s}_{\mathrm{WE}}=r_{\mathrm{WE}}-k(t) \mu_{\mathrm{NS}}+\sum_{m}\left(a_{m} \sin \Delta \phi_{m}+b_{m} \cos \Delta \phi_{m}\right) .
\end{aligned}
$$

The $k(t) \mu_{\mathrm{WE}}, k(t) \mu_{\mathrm{NS}}$ terms give the misalignment drift rates, $\mu_{\mathrm{WE}}, \mu_{\mathrm{NS}}$ being the components of the misalignment vector $\vec{\mu}=\hat{\tau}-\hat{s}$, and $k(t)$ the polhode-dependent torque coefficient, $\hat{\tau}$ is the unit vector along the spacecraft roll axis. The resonance torques are governed by the third term of Eq. (1) where $\Delta \phi_{m}(t)=\phi_{r}(t)-m \phi_{p}(t), \phi_{r}$ and $\phi_{p}$ being the known roll and polhode phases, with exact resonance occurring when the corresponding frequencies coincide, $\omega_{r}=m \omega_{p}$. To clarify the picture of a resonance, we keep only the resonance term on the right of Eq. (1).
Then the "step over" in the NS-WE plane follows, to lowest order, a Cornu spiral winding out from its initial direction a day or so before the resonance, moving across, then winding back into the new direction, its angular magnitude proportional to $\sqrt{a_{m}^{2}+b_{m}^{2}}$ [9].

Science data. Determining $r_{\mathrm{NS}}$ and $r_{\mathrm{WE}}$ requires (1) data from gyroscope, telescope, and roll reference signals (preprocessed and synchronized at $2 \mathrm{~s}$ intervals), with supporting TFM analyses based on separate $2200 \mathrm{sample} / \mathrm{s}$ gyro readout signals, and (2) the measurement model of Eq. (2) below. The data came from 11 months of science operations, where spacecraft anomalies from events such as solar-flare-induced CPU reboots resulted in 10 distinct but connectable data segments. Minor interruptions came from calibrations, transient electronics noise, and passages through the South Atlantic Anomaly.

Measurement model. With $\hat{s}, \hat{\tau}, \phi_{r}$, and $C_{g}(t)$ meanings already defined, we write SQUID signal $z(t)$ as

$$
\begin{aligned}
z(t)= & C_{g}(t)\left[\left(\tau_{\mathrm{NS}}-s_{\mathrm{NS}}\right) \cos \left(\phi_{r}+\delta \phi\right)+\left(\tau_{\mathrm{WE}}-s_{\mathrm{WE}}\right)\right. \\
& \left.\times \sin \left(\phi_{r}+\delta \phi\right)\right]+ \text { bias }+ \text { noise }
\end{aligned}
$$

$\left(\tau_{\mathrm{NS}}-s_{\mathrm{NS}}\right)$ and $\left(\tau_{\mathrm{WE}}-s_{\mathrm{WE}}\right)$ are the NS and WE projections of the misalignment $\vec{\mu}=\hat{\tau}-\hat{s}$, and $\delta \phi$ is a constant roll phase offset.

Trapped flux mapping. The information gained from trapped flux mapping was central to the three main modeling challenges [determining $C_{g}(t)$ and handling the misalignment and resonance torques]. The magnetic flux trapped in each rotor on cooling through its transition temperature formed a unique, stable fluxon pattern. By fitting to the spin harmonics of the 2200 sample/s data stream, we constructed detailed maps from which the three-dimensional orientation of each gyroscope could be tracked continuously over $\sim 2 \times 10^{9}$ turns. Rotor spin speed and spin-down rate were determined to $10 \mathrm{nHz}$ and $1 \mathrm{pHz} / \mathrm{sec}$, respectively [10]. Crucial for data analysis were (1) polhode phase $\phi_{p}(t)$ and angle $\gamma_{p}(t)$ both good to $1^{\circ}$ over the 353 days of science, and (2) detailed knowledge of the trapped flux contribution to $C_{g}(t)$.

TFM and $C_{g}(t)$. The scale factor $C_{g}(t)$ to be calibrated against aberration comes from the London moment $M_{L}$ aligned with the rotor's spin axis, plus a complex pattern of trapped flux $\sim 10^{-2} M_{L}$ fixed in its body, i.e., $C_{g}^{\mathrm{LM}}+$ $C_{g}^{\mathrm{TF}}(t)$. To meet the necessary $<10^{-4}$ accuracy for $C_{g}(t)$ required connecting data from $\sim 5200$ successive guide star valid half-orbits enabled by TFM.

TFM and resonance torques. The role of TFM in computing the resonance torque was even more remarkable. Solving Eq. (1) required precise knowledge of $\phi_{p}(t)$ and $\gamma_{p}(t)$. It is curious to reflect that if GP-B had had an ideal London moment with no trapped flux, the computation would have been impossible.

Spacecraft pointing. To model the misalignment torque, the accurate value of $\hat{\tau}(t)$ is needed continuously. 
During the half-orbit periods when the guide star is visible, it is the sum of annual and orbital aberrations and minor terms including parallax, bending of starlight by the Sun, and pointing error from the telescope signal. For the occulted periods, $\hat{\tau}(t)$ was determined from the four science gyros' SQUID signals. The connection process required fast computation of the gyro spin vector $\hat{s}(t)$ through the entire 11 months of science data.

Parameter estimator. The science data set was processed by a nonlinear batch weighted least-squares (WLS) estimator implemented in iterative sequential information filter form [11]. The cumulative information matrix for the full mission $\left(\sim 1.4 \times 10^{7}\right.$ data points per gyroscope $)$ was calculated sequentially based on one-orbit data batches. To ensure robust convergence of numerous runs with different numbers of estimated parameters, we used the sigma-point filter technique [12]. The lack of convergence observed in some runs of a standard WLS estimator was thus eliminated. Computations were critically facilitated by the replacement of differential equations (1) with their analytical solution $s_{\mathrm{NS}}(t), s_{\mathrm{WE}}(t)$ for an arbitrary $k(t)$.

Results and conclusions.-The four gyroscope signals were analyzed independently and the results combined and cross-checked in various ways. Table II lists the four relativity estimates and final joint result with $1 \sigma$ errors, also plotted as 95\% confidence ellipses in Fig. 3. The values are referenced to inertial space by correcting for the solar geodetic contribution of $7.3 \pm 0.3 \mathrm{mas} / \mathrm{yr} \mathrm{NS}$ and $-16.2 \pm 0.6 \mathrm{mas} / \mathrm{yr} \mathrm{WE}$, and for the guide star proper motion, $27.3 \pm 0.1 \mathrm{mas} / \mathrm{yr}$ NS and $-20.0 \pm$ $0.1 \mathrm{mas} / \mathrm{yr}$ WE (the latter numbers are based on the declination and right ascension rates of $27.27 \pm 0.11 \mathrm{mas} / \mathrm{yr}$ and $-20.83 \pm 0.10 \mathrm{mas} / \mathrm{yr}$, respectively, as determined in [13]).

The result for each gyroscope is the average of $10 \mathrm{drift}$ rate estimates based on 10 distinct parameter sets determined by the following uniform procedure. The submodels for scale factor $C_{g}(t)$, misalignment torque coefficient $k(t)$, polhode phase $\phi_{p}(t)$, etc., are linear combinations of certain basis functions with coefficients to be estimated. The number of terms in each submodel is increased from zero until the change in the relativistic drift rate estimates becomes less than $0.5 \sigma$. This defines the baseline set for a given gyroscope. Table II gives the weighted mean of the

TABLE II. Results

\begin{tabular}{lcc}
\hline \hline Source & $r_{\mathrm{NS}}(\mathrm{mas} / \mathrm{yr})$ & $r_{\mathrm{WE}}(\mathrm{mas} / \mathrm{yr})$ \\
\hline Gyroscope 1 & $-6588.6 \pm 31.7$ & $-41.3 \pm 24.6$ \\
Gyroscope 2 & $-6707.0 \pm 64.1$ & $-16.1 \pm 29.7$ \\
Gyroscope 3 & $-6610.5 \pm 43.2$ & $-25.0 \pm 12.1$ \\
Gyroscope 4 & $-6588.7 \pm 33.2$ & $-49.3 \pm 11.4$ \\
Joint (see text) & $-6601.8 \pm 18.3$ & $-37.2 \pm 7.2$ \\
GR prediction & -6606.1 & -39.2 \\
\hline \hline
\end{tabular}

baseline and nine "sensitivity run" estimates for each gyroscope, whereby in each run the number of terms in a single submodel is increased by 1 above the baseline value. The postfit residuals were white with $\chi^{2} \approx 1$ for all gyroscopes.

The joint four-gyro result is a weighted average of the four individual drift rates using their $2 \times 2 r_{\mathrm{NS}}, r_{\mathrm{WE}}$ subblocks of the full covariance matrix for each gyroscope. In the NS direction the scatter of the individual estimates is $28 \%$ larger than the individual uncertainties indicate, while in the WE direction it is $37 \%$ smaller. The individual and combined statistical uncertainties are corrected for their "over" and "under" dispersion using the $\chi^{2}$ of the individual estimates in the NS and WE directions. The "parameter sensitivity" covariance matrix is the scatter of all $10^{4}$ combinations of drift rate estimates obtained in 10 sensitivity runs for each of the four gyroscopes. The total covariance matrix is a sum of three matrices: the corrected statistical one, the sensitivity one, and the covariance matrix of unmodeled systematic effects. The $1 \sigma$ uncertainties of the joint result shown in Table II are the square roots of the diagonal elements of the total covariance matrix.

Table III summarizes uncertainties of the joint four-gyro result. The three main disturbances (evolving scale factor and the two Newtonian torques) are modeled and thus contribute to the statistical uncertainty. The systematic uncertainty in Table III includes (1) uncertainty from the sensitivity analysis to the number of model parameters and (2) small effects not incorporated in the model.

We performed several important data analysis crosschecks. First, the gyro drift rate results of Table II are confirmed by separate analyses of the segmented data. The 24 independent results from six data segments for each gyroscope are all consistent with the joint result within their confidence limits, demonstrating the internal

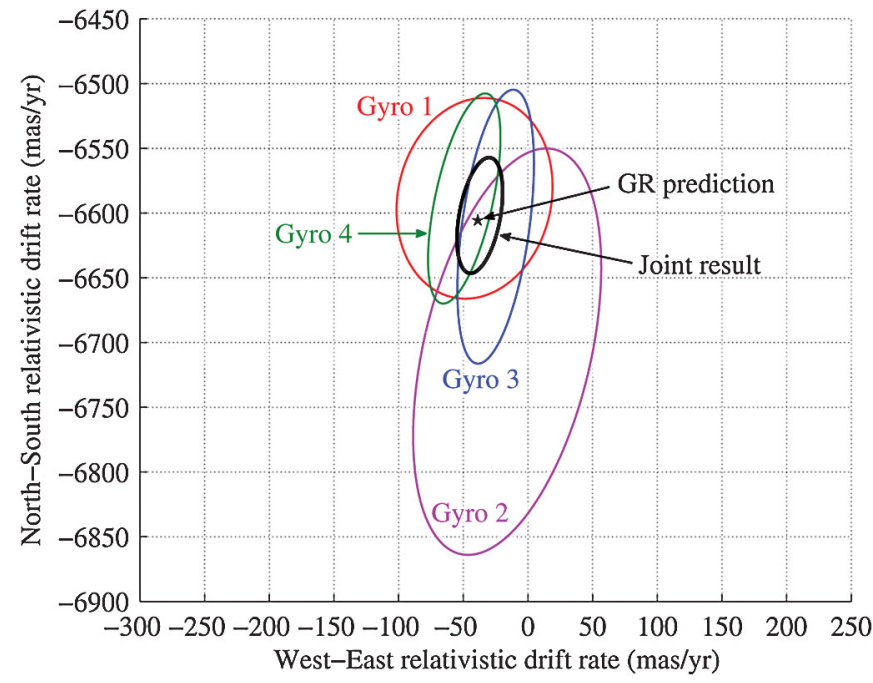

FIG. 3 (color). North-south and west-east relativistic drift rate estimates (95\% confidence) for the four individual gyroscopes (colored ellipses) and the joint result (black ellipse). 
TABLE III. Contributions to experiment uncertainty.

\begin{tabular}{lcc}
\hline \hline Contribution & NS (mas/yr) & WE (mas/yr) \\
\hline Statistical (modeled) & 16.8 & 5.9 \\
Systematic (unmodeled) & & \\
Parameter sensitivity & 7.1 & 4.0 \\
Guide star motion & 0.1 & 0.1 \\
Solar geodetic effect & 0.3 & 0.6 \\
Telescope readout & 0.5 & 0.5 \\
Other readout uncertainties & $<1$ & $<1$ \\
Other classical torques & $<0.3$ & $<0.4$ \\
Total stat + syst & 18.3 & 7.2 \\
\hline \hline
\end{tabular}

consistency of the model. Second, the misalignment torque coefficients $k$ determined during the calibration phase proved to be in excellent agreement with the end-ofmission values estimated by both algebraic and geometric analysis methods. No less impressive was the agreement between the time history $k(t)$ throughout the mission obtained by the two methods. As for the roll-polhode resonance torques, the gyro dynamics model predicts that during a resonance the gyroscope orientation axis approximately follows a Cornu spiral. Indeed, that is typically observed in orbit-by-orbit gyro orientations determined by both data analysis methods.

Further cross-checks from the geometric method included relativity estimates for 2 of the 4 gyroscopes, in both NS and WE directions, in statistical agreement with the algebraic results, and an estimate of the gravitational deflection of light by the Sun. IM Pegasi's closest approach to the Sun occurs on March 11th, with ecliptic latitude $22.1^{\circ}$ as compared with the grazing incidence value $0.265^{\circ}$ in classical light deflection tests. The maximum deflection predicted by GR is 21.7 mas; the observed $21 \pm 7$ mas serves as a neat structural confirmation of Gravity Probe B.

Lunar laser ranging has reported a measurement of the de Sitter solar geodetic effect to $0.7 \%$ [14]. Analyses of laser ranging to the LAGEOS and LAGEOS II spacecraft report a 10\%-30\% measurement of the frame-dragging effect, assuming the GR value for the geodetic precession $[15,16]$. GP-B provides independent measurements of the geodetic and frame-dragging effects at an accuracy of $0.28 \%$ and $19 \%$, respectively.

This work was supported by NASA Contract NAS839225 through Marshall Space Flight Center and by King Abdulaziz City for Science and Technology (KACST). Funding was also made available through a personal gift by Richard Fairbank with matching funds from NASA and Stanford University. A personal gift from Vance and
Arlene Coffman with matching funds by KACST was crucial to finalizing this Letter. Support from ICRA/ ICRANet is also gratefully acknowledged. GP-B would never have succeeded without the work of hundreds of people at Stanford, NASA, Lockheed Martin, and elsewhere. Some 500 graduate, undergraduate and high school students have contributed much to the project. Our special thanks go to members of the NASA-appointed Science Advisory Committee, chaired by Clifford Will, for their support and advice, and to Charbel Farhat for all his help.

*francis1@stanford.edu

[1] L. I. Schiff, Phys. Rev. Lett. 4, 215 (1960).

[2] W. de Sitter, Mon. Not. R. Astron. Soc. 77, 155 (1916).

[3] J. Lense and H. Thirring, Phys. Z. 19, 156 (1918).

[4] K.S. Thorne, in Near Zero: New Frontiers of Physics, edited by J.D. Fairbank et al. (W.H. Freeman and Co., New York, 1988), pp. 573-586.

[5] G. E. Pugh, Research memorandum 11, Weapons System Evaluation Group, the Pentagon, Washington, DC, 1959, reprinted in Nonlinear Gravitodynamics. The LenseThirring Effect., edited by R.J. Ruffini and C. Sigismondi (World Scientific, Singapore, 2003), pp. 414-426.

[6] D. B. DeBra, in Near Zero: New Frontiers of Physics, edited by J. D. Fairbank et al. (W.H. Freeman and Co., New York, 1988), pp. 691-699.

[7] J. B.Camp, T. W. Darling, and R. E. Brown, J. Appl. Phys. 69, 7126 (1991).

[8] C.C. Speake, Classical Quantum Gravity 13, A291 (1996).

[9] G. M. Keiser, J. Kolodziejczak, and A.S. Silbergleit, Space Sci. Rev. 148, 383 (2009).

[10] A. Silbergleit et al., Space Sci. Rev. 148, 397 (2009).

[11] G.J. Bierman, Factorization Methods for Discrete Sequential Estimation (Dover, New York, 2006), 2nd ed.

[12] R. van der Merwe, E. Wan, and S. J. Julier, in Proceedings of the AIAA Guidance, Navigation \& Control Conference (AIAA, Providence, RI, 2004), pp. 16-19.

[13] I. I. Shapiro et al. (to be published).

[14] J. G. Williams, X.X. Newhall, and J. O. Dickey, Phys. Rev. D 53, 6730 (1996).

[15] I. Ciufolini and E. C. Pavlis, Nature (London) 431, 958 (2004).

[16] L. Iorio, Space Sci. Rev. 148, 363 (2008).

[17] The inertial axes used in the Letter are west-east (WE) along the cross-product of the unit vector to the guide star and the unit vector $\hat{z}$ of the inertial JE2000 frame, and north-south (NS) - along the cross-product of the WE unit vector and the unit vector to the guide star. 